\title{
Timing of aneurysm surgery: the International Cooperative Study revisited in the era of endovascular coiling
}

\author{
Matthew F Lawson, ${ }^{1}$ Yueh-Yun Chi, ${ }^{2}$ Gregory J Velat, ${ }^{1}$ J D Mocco, ${ }^{1}$ Brian L Hoh ${ }^{1}$
}

${ }^{1}$ Department of Neurosurgery, University of Florida College of Medicine, Gainesville, Florida, USA

${ }^{2}$ Department of Epidemiology and Health Policy Research, University of Florida College of Medicine, Gainesville, Florida, USA

\section{Correspondence to}

Brian L Hoh, Department of Neurosurgery, University of Florida College of Medicine, PO Box 100265, Gainesville, FL 32610-0265, USA:

brian.hoh@neurosurgery.ufl.edu

Received 20 August 2009 Revised 13 December 2009

Accepted 4 January 2010

Published Online First

5 March 2010

\section{ABSTRACT}

Objective The International Cooperative Study on the Timing of Aneurysm Surgery demonstrated that subarachnoid hemorrhage (SAH) patients who underwent surgery on post-hemorrhage days 4-10 had worse outcomes than patients treated on days $0-3$ and days $11-14$. Based on these findings, it was concluded that patients who present with SAH on days 4-10 should have aneurysm surgery delayed until after day 10 . Since the study, coiling has become a treatment option and it is unclear whether these results apply to this new treatment modality. Our institution is a regional referral center for $\mathrm{SAH}$, and patients are transferred at different time points after hemorrhage. We wanted to determine whether patients that arrive on days $4-10$ were safe to undergo coiling immediately rather than waiting until after day 10 . Methods We reviewed 119 consecutive SAH patients who underwent coiling between January 2006 and June 2008. Factors of age, gender, Hunt-Hess grade, Fisher score, aneurysm size and aneurysm location were included in a regression analysis to determine the effect of day of coiling on clinical outcome at discharge.

Results Of 119 study patients, $86 \%$ had coiling on posthemorrhage days $0-3$, and $12 \%$ on days $4-10$. Patients in these cohorts did not differ in any demographic factors. Age and Hunt-Hess grade were the only predictors of mortality (age $p=0.0001$, Hunt-Hess $p=0.0110$ ) and poor outcome, defined as death or discharge to a skilled nursing facility (age $p=0.0001$, Hunt - Hess $p=0.0001$ ). Day of coiling had no effect on mortality $(p=0.5731)$ or poor outcome $(p=0.1861)$.

Conclusions Coiling of ruptured aneurysms can be performed safely on patients who arrive on posthemorrhage days $4-10$, and treatment need not be delayed after day 10, as the results of the Timing of Aneurysm Surgery Study initially suggested.

\section{INTRODUCTION}

The International Cooperative Study on the Timing of Aneurysm Surgery demonstrated that aneurysmal subarachnoid hemorrhage (SAH) patients who underwent surgery on days 4-10 post-hemorrhage had worse outcomes than patients treated on days $0-3$ or $11-14 .{ }^{12}$ One conclusion of these results is that patients who present on posthemorrhage days 4-10 should have clipping delayed until after day 10. Since the study, endovascular coil embolization has become a treatment for ruptured cerebral aneurysms and it is unclear whether the surgical results from the International Cooperative Study apply to this new treatment modality.

Our institution is a regional referral center for SAH and patients are transferred, for various reasons, at different time points after hemorrhage. While the vast majority of our patients are transferred within the first $48 \mathrm{~h}$, a significant minority arrive days later, during the suboptimal post-hemorrhage day 4-10 period. Our protocol is to treat the ruptured aneurysm, with endovascular coil embolization if possible, within $24 \mathrm{~h}$ of admission. This differs from the recommendation of the International Cooperative Study on the Timing of Aneurysm Surgery, which recommends delaying treatment until after day 10 . We reviewed our experience to determine whether it is safe to coil aneurysm patients that present on post-SAH days $4-10$.

\section{PATIENTS AND METHODS}

This study was conducted under an Institutional Review Board approved protocol in accordance with the Health Insurance Portability and Accountability Act standards for the protection of patient privacy. We reviewed a clinical database of the University of Florida neurovascular team from January 2006 through June 2008, for all patients with aneurysmal SAH who underwent endovascular coil embolization by the senior author as a primary treatment modality for a ruptured aneurysm. We recorded patient demographics and baseline clinical factors, including age, gender, aneurysm location (anterior or posterior circulation), maximal diameter, admission Hunt-Hess grade, Fisher score, post-hemorrhage day on arrival, post-hemorrhage day upon coiling and the presence of vasospasm. Discharge disposition (deceased, skilled nursing facility, rehab or home) was recorded as the primary measure of clinical outcome.

Standard SAH treatment protocols at our institution were employed for all patients. Our standard SAH treatment protocol included ventriculostomy drainage for management of hydrocephalus, oral nimodipine (60 mg every $4 \mathrm{~h}$ ) through day 21 , magnesium sulfate (12.5 g per day via continuous infusion) through day 14 and simvastatin (40 mg daily).

We grouped patients for our analysis into cohorts based on the time frames as described in the International Cooperative Study for the Timing of Aneurysm Surgery. ${ }^{2}$ Treatment cohorts included post-hemorrhage days $0-3,4-10$ and $11-21$. Statistical analyses were then performed to confirm similarity between cohort demographics, to analyze differences in discharge disposition and to identify predictors of poor outcome.

\section{Statistical analysis}

Statistical analysis was performed using SAS 9.1 (SAS Inc.). The Mantel-Haenszel test statistic for 
contingency tables was computed to determine the association between timing of coiling and mortality and discharge disposition. Multivariate logistic regression models were chosen to associate death or poor clinical outcome with important demographic and clinical variables. A type I error rate of 0.05 was used to determine the significance of all testing results.

\section{RESULTS}

From January 2006 to June 2008, 119 ruptured cerebral aneurysms in 119 patients were coiled by the senior author. The vast majority, 102 (85.7\%), underwent endovascular coiling on days $0-3,14(11.8 \%)$ underwent coiling on days $4-10$, while only 3 $(2.5 \%)$ underwent coiling on days $11-21$. Due to the small number of patients in the late treatment cohort, only those patients treated on days $0-3$ and 4-10 were directly compared. Patient demographic data and clinical characteristics of the cohorts are listed in table 1 . There were no significant differences in gender, age, aneurysm location, Hunt-Hess grade or Fisher Score between these groups. The only significant difference was average aneurysm size. Average size was $8.9 \mathrm{~mm}$ in the day $0-3$ cohort and $6.6 \mathrm{~mm}$ in the day $4-10$ cohort. This difference can be attributed to a few giant aneurysms treated on days $0-3$. The day $0-3$ cohort had 27 (26\%) aneurysms exceeding $10 \mathrm{~mm}$ in size, where the day 4-10 group had only 2 (14\%).

Mortality and discharge disposition of patients in these cohorts were similar. Of those treated on post-hemorrhage days $0-3,33 \%$ were discharged home, $32 \%$ to inpatient rehab, $9 \%$ to a skilled nursing facility and $26 \%$ died. Of those treated on posthemorrhage days 4-10, 43\% were discharged home, 14\% to rehab, $29 \%$ to a skilled nursing facility and $14 \%$ died. Table $2 \&$ figure 1 depict the mortality and discharge disposition for these cohorts.

Multivariate logistic regression analysis was performed on the outcome of death, using factors including timing of coiling, age, gender, aneurysm location, Hunt-Hess grade and Fisher score. For each factor, we computed statistical p-values ( $p$ ) and ORs with their $95 \%$ CIs from our multivariate models. For timing of coiling $(\mathrm{p}=0.6621)$, the odds of death for patients treated on days $0-3$ is 1.494 (95\% CI between 0.247 and 9.032) times the odds of

Table 1 This table displays the baseline demographics and characteristics between treatment cohorts

\begin{tabular}{|c|c|c|c|c|}
\hline \multicolumn{5}{|c|}{ Patient cohort demographics and clinical characteristics } \\
\hline & & $\begin{array}{l}\text { Coiling on } \\
\text { days } 0-3\end{array}$ & $\begin{array}{l}\text { Coiling on } \\
\text { days } 4-10\end{array}$ & p Value \\
\hline Patient No & & 102 & 14 & \\
\hline \multirow[t]{3}{*}{ Gender } & & & & 0.6878 \\
\hline & Male, n (\%) & $31(30.4)$ & $5(35.7)$ & - \\
\hline & Female, n (\%) & $71(69.6)$ & $9(64.3)$ & \\
\hline Mean age & & 58 & 53 & 0.2857 \\
\hline \multirow[t]{3}{*}{ Location } & & & & 0.2752 \\
\hline & Anterior, $\mathrm{n}(\%)$ & $65(63.7)$ & $11(78.6)$ & \\
\hline & Posterior, n (\%) & $37(36.3)$ & $3(21.4)$ & \\
\hline \multirow[t]{6}{*}{ Hunt-Hess grade, $n(\%)$} & & & & 0.8194 \\
\hline & 1 & $23(22.8)$ & $5(35.7)$ & \\
\hline & 2 & $2(2.0)$ & $0(0.0)$ & \\
\hline & 3 & $46(45.5)$ & $4(28.6)$ & \\
\hline & 4 & $30(29.7)$ & $5(35.7)$ & \\
\hline & 5 & $0(0.0)$ & $0(0.0)$ & \\
\hline \multirow[t]{5}{*}{ Fisher score, $n(\%)$} & & & & 0.7771 \\
\hline & 1 & $2(2.0)$ & $1(7.1)$ & \\
\hline & 2 & $5(4.9)$ & $0(0.0)$ & \\
\hline & 3 & $85(83.3)$ & $12(85.7)$ & \\
\hline & 4 & $10(9.8)$ & $1(7.1)$ & \\
\hline Mean size (mm) & & 8.9 & 6.6 & 0.0167 \\
\hline
\end{tabular}

Table 2 This table outlines discharge disposition of the treatment cohorts

\begin{tabular}{|c|c|c|c|c|c|}
\hline \multicolumn{5}{|c|}{ Patient mortality and discharge disposition } & \multirow[b]{3}{*}{ p Value } \\
\hline & \multicolumn{2}{|c|}{$\begin{array}{l}\text { Coiling on days } \\
0-3\end{array}$} & \multicolumn{2}{|c|}{$\begin{array}{l}\text { Coiling on days } \\
4-10\end{array}$} & \\
\hline & $\mathrm{n}$ & $\%$ & $\mathrm{n}$ & $\%$ & \\
\hline Home & 34 & 33.3 & 6 & 42.9 & \\
\hline Rehab & 33 & 32.3 & 2 & 14.3 & \\
\hline SNF & 9 & 8.8 & 4 & 28.5 & \\
\hline Deceased & 26 & 25.5 & 2 & 14.3 & \\
\hline
\end{tabular}

SNF, skilled nursing facility.

death for patients treated on days $4-10$. For age $(p=0.0004)$, a 1-year increase in age had an OR of 1.072 with 95\% CI between 1.032 and 1.115. For gender $(p=0.0447)$, female versus male had an OR of 4.098 with $95 \%$ CI of 1.034 to 16.237 . For aneurysm location ( $p=0.4268)$, anterior versus posterior had an OR of 1.644 with $95 \%$ CI of 0.483 to 5.598 . For Hunt-Hess grade ( $p=0.0396)$, a one-point increase in grade had an OR of 1.856 and $95 \%$ CI of 1.030 to 3.344. For Fisher score $(p=0.5019)$, a one-point increase in score had an OR of 1.658 and $95 \%$ CI of 0.379 to 7.259 . The factors that were significantly associated with death were advancing age and Hunt-Hess grade.

A second multivariate logistic regression analysis was performed on poor clinical outcome, defined as patients who either died or were discharged to a skilled nursing facility. Factors considered in the secondary analysis were timing of coiling, age, gender, aneurysm location, Hunt-Hess grade and Fisher score. For timing of coiling $(\mathrm{p}=0.1503)$, days $0-3$ versus days $4-10$ had an $\mathrm{OR}$ of 0.330 with $95 \%$ CI of 0.073 to 1.494 . For age $(p=0.0002)$, a 1 -year increase in age had an OR of 1.073 with $95 \%$ CI of 1.034 to 1.113 . For gender $(p=0.7858)$, female versus male had an OR of 1.154 with $95 \%$ CI of 0.411 to 3.235 . For aneurysm location $(p=0.3715)$, anterior versus posterior had an OR of 1.622 with $95 \%$ CI of 0.562 to 4.683 . For Hunt-Hess grade $(\mathrm{p}=0.0003$ ), increasing grade had an OR of 2.965 and $95 \%$ CI of 1.644 to 5.349 . For Fisher score $(\mathrm{p}=0.8573)$, increasing score had an OR of 1.125 and $95 \%$ CI of 0.313 to 4.043 . Both age and Hunt-Hess grade were significant factors associated with poor outcome.

\section{DISCUSSION}

Timing of aneurysm surgery after SAH has been a controversial issue in neurosurgery since the late 1960s. ${ }^{3}$ In 1969, Sahs ${ }^{4}$

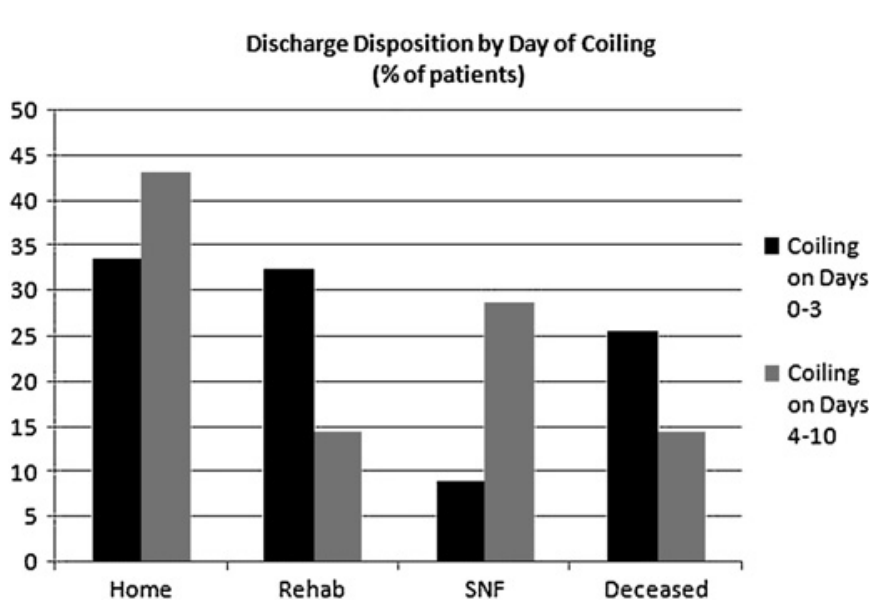

Figure 1 Discharge disposition by treatment cohort; SNF, skilled nursing facility. 
established craniotomy for aneurysm clipping or ligation as a superior treatment to bedrest and medical therapy for aneurysm rupture. Debate over the relative merits of early or late surgical intervention persisted until the publication of the International Cooperative Study on the Timing of Aneurysm Surgery Trial, conducted in the 1980s. ${ }^{3}$ This observational study of 3521 patients with ruptured intracranial aneurysms demonstrated no difference in outcome between patients who underwent early surgery, post-hemorrhage days $0-3$, compared with late surgery, days 11-14. Patients who underwent surgery in the intermediate period, especially days 7-10, had worse outcomes when compared with the early and late groups. ${ }^{2}$ Several recent observational studies have had similar results and have supported the finding that early versus late surgical intervention have equivalent outcomes. ${ }^{5} 6$

What has not been well studied in the literature is whether or not timing of endovascular coiling has implications on outcome. There has been increasing utilization of endovascular coil embolization for treatment of ruptured aneurysms over the past two decades. The only large review of the timing of endovascular treatment of aneurysms, by Baltsavius ${ }^{7}$ in 2000, demonstrated that timing of coiling did not affect 6-month outcome. Recently, the International Subarachnoid Aneurysm Trial (ISAT) demonstrated that for patients who could undergo either surgical clipping or endovascular coiling for a ruptured aneurysm, those who had coiling were more likely to be independent at both 1 and 7 years post-hemorrhage. ${ }^{8}$

Our protocol is to treat ruptured aneurysms within $24 \mathrm{~h}$ of admission, and we do not advocate delayed treatment of intracranial aneurysms. Our institution is a regional referral center for SAH and a single neurovascular team offers both surgical clipping and endovascular coil embolization. We have had a number of patients who either presented, were transferred to our facility or were stabilized for coiling on post-hemorrhage days 4-10. Our goal for this study was to show that endovascular treatment during this 'high-risk' period is safe and that aneurysm coiling should not be delayed until after day 10 .

In this retrospective study of 119 patients who underwent coiling of a ruptured aneurysm, there was no significant difference in age, gender, aneurysm location (anterior or posterior circulation), pretreatment Hunt-Hess grade or Fisher score between those coiled on post-hemorrhage days $0-3$ and those treated on days $4-10$. The only significant difference in preprocedure medical characteristics was the larger aneurysm size in the early treatment cohort $(8.9 \mathrm{~mm}$ vs $6.6 \mathrm{~mm})$. We feel this is attributable to the presence of 27 aneurysms in excess of $10 \mathrm{~mm}$ in this group that skewed the average size of the cohort. If the outlying data points of the giant aneurysms are excluded, the average size of the two groups is not significantly different.

We found that mortality and discharge disposition in the cohort treated on days $0-3$ was not statically different from those treated on days 4-10. They had similar rates of death, discharge to nursing facilities, rehab and home. Multivariate regression analyses did not find timing of coiling (either days $0-3$ or days 4-10) to be a predictor of death or poor outcome. Regression analyses found both age and Hunt-Hess grade to be significant predictors of death and poor outcome. Our results suggest that coiling on post-hemorrhage days 4-10 does not correlate with increased mortality or worsened discharge disposition.

There was a significantly higher rate of vasospasm at the time of treatment for those patients treated on days 4-10 when compared with the day $0-3$ cohort. Five of 14 patients $(36 \%)$ in the day 4-10 group had vasospasm at the time of coiling, while only 5 of 103 patients (5\%) in the day $0-3$ group had evidence of radiographic spasm at the time of treatment. Of the five patients in the day 4-10 cohort that presented with vasospasm at the time of treatment, all five were treated with intra-arterial verapamil and one required balloon angioplasty for treatment at the time of coiling. A significant proportion of the morbidity in the day 4-10 group was associated with vasospasm at the time of treatment. Of the five patients in this group who had vasospasm, only one improved to the point of being discharged home, one died and three required long-term skilled nursing care or longterm rehab care. It appears that those patients who arrive during the day 4-10 period who are in moderate to severe vasospasm have a trend toward worse outcomes than those who are not in vasospasm at the time of treatment. Nevertheless, if those patients who presented in vasospasm did not undergo coiling at the time of presentation, it is likely that they would have succumbed to vasospasm. By treating these patients immediately we were able to diagnose and perform treatment for vasospasm at the time of aneurysm coiling.

Several weaknesses are inherent in this study. First, it is retrospective in nature and may therefore contain selection bias that is not immediately apparent. Second, we acknowledge that other factors throughout the hospital course of a SAH patient may contribute to morbidity and mortality. We did not attempt to record or analyze stroke rate, ICU complications, hydrocephalus, presence of external ventricular drain, seizures or need for ventriculoperitoneal shunt placement. Third, our sample size was small, which may limit our ability to detect a difference if one truly exists.

\section{CONCLUSIONS}

Endovascular coil embolization of ruptured intracranial aneurysms may be performed safely on patients who arrive on post-hemorrhage days 4-10, without significant increase in mortality or poor outcome and despite a higher rate of vasospasm at the time of treatment. Coil embolization need not be delayed until after day 10, as the results of the Timing of Aneurysm Surgery Study initially suggested for surgical clipping. For

\section{Key messages}

- The International Cooperative Study on the Timing of Aneurysm Surgery concluded that patients that present with subarachnoid hemorrhage (SAH) on post-hemorrhage days 4-10 should have aneurysm surgery delayed until after day 10.

- Since the study, coiling has become a treatment option and it is unclear whether these surgical results apply to aneurysm coiling, particularly for patients who arrive at regional SAH referral centers during the suboptimal post-hemorrhage days $4-10$.

- We reviewed 119 consecutive SAH patients treated at our facility and conducted a regression analysis to determine the effect of day of coiling on clinical outcome at discharge.

- Age and Hunt-Hess grade were the only predictors of mortality and poor outcome, and day of coiling (days $0-3$ versus days $4-10$ ) had no effect on mortality or poor outcome.

- Coiling of ruptured aneurysms can be performed safely on patients who arrive on post-hemorrhage days 4-10, and endovascular treatment need not be delayed until after day 10. 
patients who present on days 4-10 with vasospasm, immediate coiling provides the opportunity to diagnose and treat vasospasm at the time of the coiling procedure. The only predictors of poor outcome and death in SAH patients who underwent coiling were age and Hunt-Hess grade.

Competing interests JDM serves as a consultant for both Codman Neurovascular and Actelion Pharmaceuticals. He is also a consultant for Nfocus Neuromedical Inc. and has equity options.

BLH serves as a consultant for both Micrus Endovascular and Codman Neurovascular. He has also received educational grants from both Micrus and Codman. Others: None.

Ethics approval This study was conducted with the approval of the University of Florida Institutional Review Board (IRB-01).

Contributors Concepts and Design: MFL, BLH. Intellectual Contribution: All authors. Data Collection: MFL. Data Review \& Interpretation: All authors. Manuscript Preparation: MFL, BLH. Manuscript Review: All authors.

Provenance and peer review Not commissioned; externally peer reviewed.

\section{REFERENCES}

1. Kassell NF, Torner JC. The International Cooperative study on timing of aneurysm surgery. Acta Neurochir (Wien) 1982:63:119-23.

2. Kassell NF, Torner JC, Jane JA, et al. The International Cooperative Study on the timing of aneurysm surgery. Part 2: surgical results. J Neurosurg 1990:73:37-47.

3. Stein SC. Brief history of surgical timing: surgery for ruptured intracranial aneurysms Neurosurg Focus 2001;11:E3.

4. Sahs AL. Intracranial aneurysms and subarachnoid hemorrhage; a cooperative study. Philadelphia: Lippincott, 1969.

5. Nieuwkamp DJ, de Gans K, Algra A, et al. Timing of aneurysm surgery in subarachnoid haemorrhage - an observational study in The Netherlands. Acta Neurochir (Wien) 2005;147:815-21

6. Ross N, Hutchinson PJ, Seeley H, et al. Timing of surgery for supratentorial aneurysmal subarachnoid haemorrhage: report of a prospective study. J Neurol Neurosurg Psychiatr 2002; 72:480-4.

7. Baltsavias GS, Byrne JV, Halsey J, et al. Effects of timing of coil embolization after aneurysmal subarachnoid hemorrhage on procedural morbidity and outcomes. Neurosurgery 2000;47:1320-9; discussion 29-31.

8. Molyneux AJ, Kerr RS, Yu LM, et al. International subarachnoid aneurysm trial (ISAT) of neurosurgical clipping versus endovascular coiling in 2143 patients with ruptured intracranial aneurysms: a randomised comparison of effects on survival, dependency, seizures, rebleeding, subgroups, and aneurysm occlusion. Lancet 2005;366:809-17. 